\title{
Structural Synthesis of Multi-Fingered Hands
}

Department of Mechanical Engineering, National Taiwan University,

Taipei, Taiwan 106 e-mail: jjlee@ccms.ntu.edu.tw

Lung-Wen Tsai

Fellow ASME

Department of Mechanical Engineering, Bourns College of Engineering,

University of California,

Riverside, CA 92521

e-mail: Iwtsai@engr.ucr.edu
The kinematic structure of a mechanical hand typically resembles the skeleton of a human hand and it is usually designed to emulate a subset of the functions of a human hand. This paper analyzes the structural characteristics associated with the functional requirements of a mechanical hand. Using the mobility criterion and force closure concept, constraint equations for structural synthesis of multi-fingered mechanical hands are derived. A procedure for systematic enumeration of the kinematic structures of mechanical hands is established. An atlas of feasible kinematic structures of multi-fingered hands having a mobility number ranging from 3 to 6 and contact degrees of freedom ranging from 1 to 5 is developed. Further, two theorems concerning the structural characteristics of mechanical hands are presented. [DOI: 10.1115/1.1467080]

Keywords: Structural Characteristics, Structural Synthesis, Mobility, Mechanical Hands

\section{Introduction}

The kinematic structure of a robotic manipulator generally employs a serial kinematic chain attached with an end-effector or gripper capable of simple gripping. The use of such structure has limited functions, e.g., unable to provide fine motion or force control. To improve the dexterity and achieve fine manipulation, anthropomorphic hands with multiple fingers have been developed.

Most mechanical hands were designed to have a plurality of fingers, each with a number of joints, to emulate some of the functions of human hands [1]. In this way, the kinematic structure of mechanical hands usually resembles the skeleton of a human hand. Several multi-fingered mechanical hands can be found in the literature, to name a few, the mechanical hand developed by Skinner [2], the multi-fingered hand designed by Okada [3], the Stanford/JPL hand (or Salisbury hand) [4], the Utah-MIT hand [5], and the NTU-1 hand by National Taiwan University [6]. However, most of these mechanical hands were constructed by the designers' intuition or by emulating grasping patterns of human hands. Relatively few articles dealing with the structure synthesis of mechanical hands can be found in the literature.

The structural synthesis of mechanical hands involves a determination of the number of fingers, number of links and joints in each finger, and the types of contact between the finger and an object to be manipulated. Salisbury and Roth [7] performed a structural synthesis of hand mechanisms having up to three fingers. Then they developed a three-fingered hand with three joints on each finger [8]. Except for this, not much literature dealing with the design of multi-fingered hands from the kinematic structure point of view can be found.

A field that is closely related to the structural synthesis of multifingered hands is the structural kinematics of parallel manipulators. However, there is a distinct difference between the two. As shown in Fig. 1(a) and (b), the kinematic pairs formed between the fingers and a grasped object in a multi-fingered hand are fundamentally different from that formed between the legs and the end-effector of a parallel manipulator. An object is said to be under force closure if the constraints provided by a mechanical manipulator can resist only certain disturbance forces acting on the body, and it is said to be under form closure if it can resist arbitrary disturbance forces $[9,10]$. The grasp shown in Fig. 1(a) is said to be under force closure, since each finger can only push but

Contributed by the Mechanisms and Robotics Committee for publication in the JoURNAL OF MECHANICAL DESIGN. Manuscript received Apr. 2001. Associate Editor: M. Raghavan. not pull the object at the point of contact. A secured grasp depends to a large extent on the directions and locations of the contact forces provided by the fingers. On the other hand, the end effector shown in Fig. 1(b) is said to be under form closure, since the constraints imposed on the end effector by the legs can resist arbitrary disturbance forces once a proper number of the joints are locked. A number of articles on the structural synthesis of parallel manipulators can be found [11-14]. Hunt [12] applied the theory of screw systems for type synthesis of parallel manipulators. Tsai [14] made a classification of the kinematic structures of parallel manipulators. These works led us to a structure synthesis of the kinematic structure of multi-fingered hands.

First, the contact freedom between a finger and an object is reviewed. Then, the mobility criterion and other constraint equations related to the contact freedom, the number of joints and links in a finger, and the number of fingers are established. A systematic procedure for the enumeration of the kinematic structures is developed from which an atlas of feasible kinematic structures of multi-fingered mechanical hands is produced. Finally, two theorems concerning the structure characteristics of mechanical hands are derived.

\section{Contact Geometry}

A rigid body without any constraint possesses six degrees of freedom (DOF) in three-dimensional space. When the body is brought into contact with another rigid body, the motion of the body becomes somewhat restricted. The degree to which the motion of the body is restricted depends on the nature of contact, including the pairing geometry, the frictional restraint, and the location on which the contact is applied $[4,11]$. The relative DOF between two contacting bodies, with or without frictional restraint, can be summarized in Table 1. It can be seen that there is every possible physical pairing to generate contact degrees of freedom from zero to five. This result will be used in the following sections for structural analysis.

\section{Structural Characteristics}

3.1 Assumptions. When a multi-fingered hand grasps an object, it forms an equivalent closed-loop mechanism as shown in Fig. 1(a). The following assumptions are made:

- The contact between the mechanical hand and an object occur at the distal link of all fingers.

- All contact geometries are of the same type.

- All joints are one-DOF joints. 


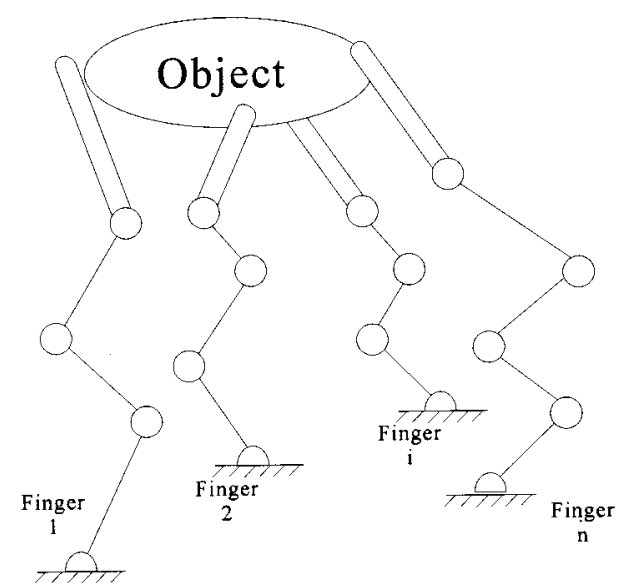

(a)

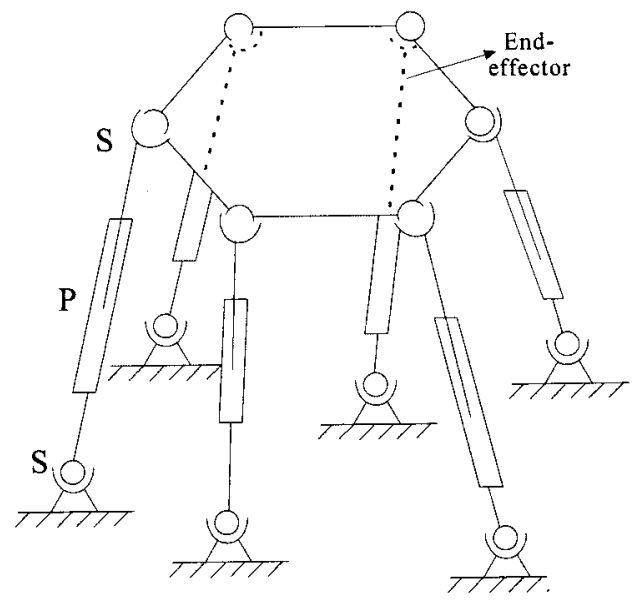

(b)

Fig. 1 A mechanical hand and a parallel manipulator: (a) mechanical hand; (b) parallel manipulator

3.2 Mobility Equation. Let $F$ be the mobility of a handobject system, $n$ the number of the fingers, $m_{i}$ the number of links which is equal to the number of the joints in the $i^{\text {th }}$ finger, and $f$ the contact DOF between the finger and an object. Then, the total number of links in the hand-object system, including the object but not the fixed base, is equal to $\left(2+\sum_{i=1}^{n} m_{i}-1\right)$, the number of constraints imposed by the joints in all fingers is equal to $5 \sum_{i=1}^{n} m_{i}$, and the number of constraints imposed by the contact points is equal to $n(6-f)$. Hence, the mobility of the resulting hand-object system is given by

$$
F=6\left(\left(2+\sum_{i=1}^{n} m_{i}\right)-1\right)-\left(5 \sum_{i=1}^{n} m_{i}+n(6-f)\right) .
$$

After simplification, Eq. (1) can be written as

$$
F=6+\sum_{i=1}^{n} m_{i}-n(6-f)
$$

Table 1 Contact degrees of freedom versus contact geometry.

\begin{tabular}{lcccc}
\hline \hline Type of Contact & Point & Line & Plane & Soft finger [4] \\
\hline Without Friction & 5 & 4 & 3 & 2 \\
With Friction & 3 & 1 & 0 & - \\
\hline \hline
\end{tabular}

Table 2 Minimum number of fingers as a function of contact degrees of freedom.

\begin{tabular}{llllll}
\hline \hline$f$ & 1 & 2 & 3 & 4 & 5 \\
$n_{\min }$ & 2 & 2 & 3 & 4 & 7 \\
\hline \hline
\end{tabular}

Equation (2) is useful for calculating the mobility of a system formed by a mechanical hand and an object.

For a mechanical hand-object system with $F$ degrees of freedom, generally only $F$ variables in the system can be controlled independently. For direct kinematics, only $F$ joint angles in the fingers can be controlled independently and the remaining joint angles are determined by the constraints imposed by the joints and contact points. For the inverse kinematics, only $F$ parameters associated with the position and orientation of the grasped object can be specified independently. In this regard, the mobility number serves as a criterion for dexterous manipulation. The more dexterity a mechanical hand is desired, the greater the mobility number is needed. Yet, the converse statement does not necessarily hold as will be discussed later.

Using Eq. (2), the number of fingers, $n$, and the number of links (and joints), $m_{i}$, for each finger can be determined as functions of the mobility number, $F$, and the contact degrees of freedom, $f$. It is possible to find for all the combinations by an exhaustive search of the design parameters. However, it would be more efficient if additional constraints can be developed prior to the search.

3.3 Number of Fingers. Equation (2) provides only a criterion for counting the mobility of a mechanical hand-object system. However, it is not a sufficient condition for predicting whether a grasped object can be manipulated as desired. To determine whether a mechanical hand can completely immobilize an object, one must further count the constraints exerted on the object by the fingers.

The concept of force closure of a mechanical system was first introduced by Reuleaux [9]. Reuleaux pointed out that at least four frictionless point contacts are required to completely immobilize an object in two-dimensional space. Somov [15] and later Lakshminarayana [10] showed that at least seven frictionless contact points are required to immobilize an object in threedimensional space. Recently, Rimon and Burdick [16] and Yoshikawa [17] showed that an object of special geometry can be immobilized by a number of contacts that is fewer than that predicted by Reuleaux, Somov, and Lakshminarayana. The results of Reuleaux and Lakshminarayana can be interpreted by the theory of screws. Each constraint provided by a contact can be viewed as a wrench vector exerted on the body. It is well known that an $\mathrm{n}$-dimensional vector space requires at least $\mathrm{n}+1$ vectors to form a closure [18]. Since a rigid body has six degrees of freedom in space, at least seven wrenches are needed to completely immobilize an object. Hence, we require the total number of constraints, including frictional constraints, imposed on the object by all fingers to be at least equal to 7. Assuming that each finger comes in contact with the object at only one point and $f$ is the degrees of freedom at the point of contact, the above statement can be written as

$$
n(6-f) \geqslant 7,
$$

Solving Eq. (3) for $n$, we obtain

$$
n \geqslant 7 /(6-f) .
$$

Given the type of contact, $f$, Eq. (4) establishes the lower bound for the number of fingers needed in a mechanical hand to grasp an object securely in space. Solving Eq. (4) yields the minimum number of fingers as a function of the contact degrees of freedom as listed in Table 2 .

The actual number of fingers on a hand is often determined from practical point of view. In this paper, we limit ourselves to 


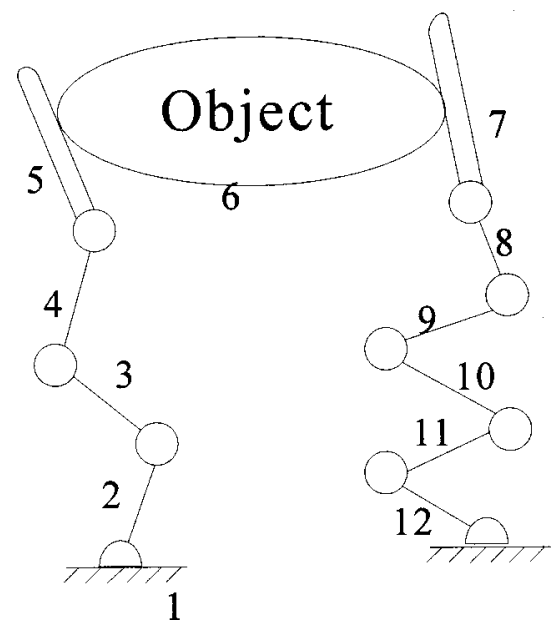

Fig. 2 A 6-DOF hand-object system for which the object cannot possess 6-DOF motion

those kinematic structures having at least 3 and no more than 7 fingers, since it is trivial to study a mechanical hand with only one or two fingers and too complex for a mechanical hand with more than 7 fingers. Thus, we artificially impose the condition:

$$
7 \geqslant n \geqslant 3 \text {. }
$$

In this regard, the upper bound on the number of fingers is always equal to 7 whereas the lower bound is equal to 3 or $n_{\text {min }}$ listed in Table 2 whichever is larger.

3.4 Number of Joints in a Finger. The connectivity, $C_{i}$, of a finger is defined as the sum of the degrees of freedom in all the joints, including the contact point, of the finger. Specifically,

$$
C_{i}=m_{i}+f
$$

For a mechanical system with an overall mobility $F$, the end effector may not necessarily possess $F$ degrees of freedom. For example, if the contact degree of freedom is equal to 1 and all the joints in the two-fingered hand shown in Fig. 2 are 1-DOF joints, the overall hand-object system has 6 degrees of freedom. However, the object cannot be manipulated freely in 3-dimensional space because its motion is constrained by the left finger of connectivity 5 . Hence, it is necessary to provide each finger with a sufficient connectivity number such that the object can be manipulated as desired.

It is obvious that for a grasped object to be manipulated with $F$-DOF motion the connectivity of each finger must be at least equal to $F$. Furthermore, the connectivity should not exceed 6 to avoid redundancy in connectivity [14]. Hence, we impose the following condition:

$$
6 \geqslant C_{i} \geqslant F \text {. }
$$

Substituting Eq. (6) into (7) and after rearrangement, we obtain

$$
6-f \geqslant m_{i} \geqslant F-f \text {. }
$$

Equation (8) determines the lower and upper bounds on the number of joints (and links) required for each finger as a function of the mobility number and contact degrees of freedom. In general, the desired mobility of a grasped object is determined by a given application. This is equivalent to a product specification in the conceptual design phase of a machine. In this paper, we limit ourselves to

$$
6 \geqslant F \geqslant 3 \text {. }
$$

\section{Structural Synthesis}

The kinematic structure of a mechanical hand should satisfy the conditions imposed by Eqs. (2), (4), (5), and (8). Using these equations, a systematic procedure can be developed for the enumeration of feasible kinematic structures of multi-fingered hands. In the following, we use a three-DOF system to illustrate the procedure.

Step 1. Determine the mobility number, $F$, for the hand-object systems of interest. In this example, we let $F=3$.

Step 2. Select a contact type. Depending on the type of contact, the contact degrees of freedom, $f$, can assume the values of 1 to 5 as outlined in Table 1.

Step 3. Determine the lower bound on the number of fingers by applying Eqs. (4) and (5). For example, given $F=3$ and $f=1$, we obtain $n_{\min }=2$ from Eq. (4) or Table 2. However, we let $n_{\min }=3$ due to the condition imposed by Eq. (5). According to Eq. (5), the upper bound is always equal to 7 .

Step 4. For a given number of fingers, $n$, determine the number of joints (and links), $m_{i}$, in each finger by solving Eq. (2) subject to the constraint imposed by Eq. (8). For example, for $F=3, f$ $=1$, and $n=3$, Eqs. (2) and (8) reduce to

$$
\begin{gathered}
m_{1}+m_{2}+m_{3}=12, \\
5 \geqslant m_{i} \geqslant 2 .
\end{gathered}
$$

Therefore, we can solve Eq. (10) for positive integers of $m_{i}$ by varying the values of $m_{i}$ 's from 2 to 5 . A simple nested do-loop program can be written to find all the solutions of $m_{i}$ ' $s$. Without loss of generality, we add the condition that $m_{1} \leqslant m_{2} \leqslant m_{3}$. As a result, we obtain three feasible solutions: $\left(m_{1}, m_{2}, m_{3}\right)=(2,5,5)$, $(3,4,5)$, and $(4,4,4)$.

Step 5. Repeat Step 4 for a different value of $n$ until all the feasible values of $n$ that fall between the lower and upper bounds are accounted for.

Step 6. Repeat Step 2 for a different type of contact, $f$, until all the possible types of contact are exhausted.

Step. 7. Repeat Step 1 for a different mobility number.

Using the above procedure, all feasible structures of multifingered hands with the mobility number ranging from 3 to 6 and the contact degrees of freedom ranging from 1 to 5 have been enumerated. Table 3 shows the feasible kinematic structures as a function of the mobility number, $F$, contact degrees of freedom, $f$, and number of fingers, $n$.

We noted that the kinematic structure of the MIP2 gripper [19] is given by $\left(m_{1}, m_{2}, m_{3}, m_{4}, m_{5}, m_{6}, m_{7}\right)=(1,1,1,1,1,1,1)$. Assuming that all contact points are frictionless, $f=5$, then the resulting hand-object system will possess six degrees of freedom. Similarly, the kinematic structure of Salisbury's hand [4] is given by $\left(m_{1}, m_{2}, m_{3}\right)=(3,3,3)$. Therefore, if all three fingers are in contact with an object at appropriate locations with friction, $f$ $=3$, the resulting hand-object system will also possess six degrees of freedom. However, if the fingers are soft, $f=2$, the resulting hand-object system will have only three degrees of freedom. The kinematic structure of the NTU hand [6] is given by $\left(m_{1}, m_{2}, m_{3}, m_{4}, m_{5}\right)=(3,3,3,4,4)$. Hence, if all fingers are in contact with an object with friction, $f=3$, the resulting handobject system will possess six degrees of freedom with some redundancy in connectivity. On the other hand, if the fingers are soft, $f=2$, the resulting hand-object system will possess only three degrees of freedom.

\section{Symmetrical Structural Topology}

A mechanical hand is said to be symmetrical if all the fingers share the same structural topology. Specifically, the contact degrees of freedom, the number of joints, and the number of links are the same for all fingers [14]. Feasible mechanical hands with symmetrical finger topology are already included in Table 3 . It can be seen that symmetrical hands exist only for those mechanisms 
Table 3 Feasible kinematic structures of mechanical hands.

\begin{tabular}{|c|c|c|c|c|c|c|}
\hline \multirow[b]{2}{*}{ Mobility, F } & \multirow{2}{*}{$\begin{array}{l}\text { Contact } \\
\text { freedom, f }\end{array}$} & \multicolumn{5}{|c|}{ Feasible kinematic structures of multi-fingered hands, $\left(\mathrm{m}_{1}, m_{2}, ..\right)$} \\
\hline & & $\mathrm{n}=3$ & $\mathrm{n}=4$ & $\mathrm{n}=5$ & $\mathrm{n}=6$ & $\mathrm{n}=7$ \\
\hline \multirow[t]{10}{*}{3} & 1 & $(2,5,5)$ & $(2,5,5,5)$ & $(2,5,5,5,5)$ & $(2,5,5,5,5,5)$ & $(2,5,5,5,5,5,5)$ \\
\hline & & $(3,4,5)$ & $(3,4,5,5)$ & $(3,4,5,5,5)$ & $(3,4,5,5,5,5)$ & $(3,4,5,5,5,5,5)$ \\
\hline & & $(4,4,4)$ & $(4,4,4,5)$ & $(4,4,4,5,5)$ & $(4,4,4,5,5,5)$ & $(4,4,4,5,5,5,5)$ \\
\hline & 2 & $(1,4,4)$ & $(1,4,4,4)$ & $(1,4,4,4,4)$ & $(1,4,4,4,4,4)$ & $(1,4,4,4,4,4,4)$ \\
\hline & & $(2,3,4)$ & $(2,3,4,4)$ & $(2,3,4,4,4)$ & $(2,3,4,4,4,4)$ & $(2,3,4,4,4,4,4)$ \\
\hline & & $(3,3,3)$ & $(3,3,3,4)$ & $(3,3,3,4,4)$ & $(3,3,3,4,4,4)$ & $(3,3,3,4,4,4,4)$ \\
\hline & 3 & $(1,2,3)$ & $(1,2,3,3)$ & $(1,2,3,3,3)$ & $(1,2,3,3,3,3)$ & $(1,2,3,3,3,3,3)$ \\
\hline & & $(2,2,2)$ & $(2,2,2,3)$ & $(2,2,2,3,3)$ & $(2,2,2,3,3,3)$ & $(2,2,2,3,3,3,3)$ \\
\hline & 4 & - & $(1,1,1,2)$ & $(1,1,1,2,2)$ & $(1,1,1,2,2,2)$ & $(1,1,1,2,2,2,2)$ \\
\hline & 5 & - & - & - & - & - \\
\hline \multirow[t]{8}{*}{4} & 1 & $(3,5,5)$ & $(3,5,5,5)$ & $(3,5,5,5,5)$ & $(3,5,5,5,5,5)$ & $(3,5,5,5,5,5,5)$ \\
\hline & & $(4,4,5)$ & $(4,4,5,5)$ & $(4,4,5,5,5)$ & $(4,4,5,5,5,5)$ & $(4,4,5,5,5,5,5)$ \\
\hline & 2 & $(2,4,4)$ & $(2,4,4,4)$ & $(2,4,4,4,4)$ & $(2,4,4,4,4,4)$ & $(2,4,4,4,4,4,4)$ \\
\hline & & $(3,3,4)$ & $(3,3,4,4)$ & $(3,3,4,4,4)$ & $(3,3,4,4,4,4)$ & $(3,3,4,4,4,4,4)$ \\
\hline & 3 & $(1,3,3)$ & $(1,3,3,3)$ & $(1,3,3,3,3)$ & $(1,3,3,3,3,3)$ & $(1,3,3,3,3,3,3)$ \\
\hline & & $(2,2,3)$ & $(2,2,3,3)$ & $(2,2,3,3,3)$ & $(2,2,3,3,3,3)$ & $(2,2,3,3,3,3,3)$ \\
\hline & 4 & - & $(1,1,2,2)$ & $(1,1,2,2,2)$ & $(1,1,2,2,2,2)$ & $(1,1,2,2,2,2,2)$ \\
\hline & 5 & - & - & - & - & - \\
\hline \multirow[t]{5}{*}{5} & 1 & $(4,5,5)$ & $(4,5,5,5)$ & $(4,5,5,5,5)$ & $(4,5,5,5,5,5)$ & $(4,5,5,5,5,5,5)$ \\
\hline & 2 & $(3,4,4)$ & $(3,4,4,4)$ & $(3,4,4,4,4)$ & $(3,4,4,4,4,4)$ & $(3,4,4,4,4,4,4)$ \\
\hline & 3 & $(2,3,3)$ & $(2,3,3,3)$ & $(2,3,3,3,3)$ & $(2,3,3,3,3,3)$ & $(2,3,3,3,3,3,3)$ \\
\hline & 4 & - & $(1,2,2,2)$ & $(1,2,2,2,2)$ & $(1,2,2,2,2,2)$ & $(1,2,2,2,2,2,2)$ \\
\hline & 5 & - & - & - & - & - \\
\hline \multirow[t]{5}{*}{6} & 1 & $(5,5,5)$ & $(5,5,5,5)$ & $(5,5,5,5,5)$ & $(5,5,5,5,5,5)$ & $(5,5,5,5,5,5,5)$ \\
\hline & 2 & $(4,4,4)$ & $(4,4,4,4)$ & $(4,4,4,4,4)$ & $(4,4,4,4,4,4)$ & $(4,4,4,4,4,4,4)$ \\
\hline & 3 & $(3,3,3)$ & $(3,3,3,3)$ & $(3,3,3,3,3)$ & $(3,3,3,3,3,3)$ & $(3,3,3,3,3,3,3)$ \\
\hline & 4 & - & $(2,2,2,2)$ & $(2,2,2,2,2)$ & $(2,2,2,2,2,2)$ & $(2,2,2,2,2,2,2)$ \\
\hline & 5 & - & - & - & - & $(1,1,1,1,1,1,1)$ \\
\hline
\end{tabular}

having 3 or 6 degrees of freedom of dexterity. We note that all the 6-DOF mechanical hands are symmetrical. This fact can be proved as follows.

Let $m$ be the number of links (and joints) and $C$ the connectivity of a finger. Replacing each $m_{i}$ in Eq. (2) by $m$ and making use of Eq. (6), we obtain

$$
F=6+n(C-6) \text {. }
$$

Substituting $F=6$ into Eq. (12), we obtain

$$
n(C-6)=0 \text {. }
$$

Since $n$ is a positive integer, the only non-trivial solution to Eq. (13) is $C=6$, regardless of the number of fingers. This leads to the following theorems.

Theorem 1: Adding or removing a finger of connectivity 6 to a mechanical hand has no effect on the mobility of the resulting hand-object system.

Proof: Assume that a finger with the same contact degrees of freedom is added onto a mechanical hand with $n$ fingers. Applying Eq. (2), the mobility of the resulting mechanism is given by

$$
F^{\prime \prime}=6+\sum_{i=1}^{n+1} m_{i}-(n+1)(6-f) .
$$

Subtracting Eq. (14) from (2) and after simplification, we obtain

$$
F^{\prime \prime}=F-6+\left(m_{n+1}+f\right)=F-\left(6-C_{n+1}\right) .
$$

It can be concluded that, in general, the addition of a finger reduces the mobility of the resulting hand-object system by ( 6 $\left.-C_{n+1}\right)$. However, if a finger with a connectivity of $C=6$ is added to the hand, Eq. (15) gives $F^{\prime}=F$. Therefore, the addition of a finger with $C=6$ does not change the mobility of the resulting hand-object system.

Likewise, the mobility of a hand-object system after removing a finger is given by

$$
F^{\prime}=6+\sum_{i=1}^{n-1} m_{i}-(n-1)(6-f) .
$$

Subtracting Eq. (16) from (2) and after simplification, we obtain

$$
F^{\prime \prime}=F+6-\left(m_{n}+f\right)=F+\left(6-C_{n}\right) .
$$

Hence, the removal of a finger increases the mobility of the resulting hand-object system by $\left(6-C_{n}\right)$. However, if a finger with $C=6$ is removed, Eq. (17) gives precisely $F^{\prime \prime}=F$. This completes the proof of Theorem 1 .

Theorem 2: For a 6-DOF mechanical hand, all fingers must have a connectivity of 6 and, therefore, is symmetrical.

Proof: we prove this by deduction. It is obvious that the kinematic structure for a one-finger system of mobility 6 is a finger of connectivity of $C=6$. According to Theorem 1 , adding a $C=6$ finger to the one-finger system does not affect the mobility of the resulting two-finger system. Hence, starting from a one-finger hand of mobility 6 , it is necessary to add a finger of identical structural topology to form a two-finger hand. Similarly, a threefinger hand is obtained by adding a third finger of identical structural topology, and so on.

\section{Conclusion}

The structural characteristics of mechanical hands have been investigated. Using these characteristics, a systematic procedure for the enumeration of the kinematic structures of mechanical hands is established. An atlas of feasible kinematic structures of mechanical hands having a mobility number ranging from 3 to 6 and contact degrees of freedom ranging from 1 to 5 is developed. Finally, two theorems related the structural characteristics of mechanical hands are derived. This work may be helpful for the design of mechanical hands during the conceptual design phase.

\section{Nomenclature}

$C=$ connectivity of a finger in a symmetrical hand

$C_{i}=$ connectivity of the $i^{\text {th }}$ finger

$F=$ mobility of a hand-object system

$f=$ contact freedom between a finger and an object

$m=$ number of joints (and links) in a finger of a symmetrical hand

$m_{i}=$ number of joints (and links) in the $i^{\text {th }}$ finger

$n=$ number of the fingers 


\section{References}

[1] Pham, D. T., and Heginbotham, W. B., 1986, Robot Grippers, IFS (Publications) Ltd, UK.

[2] Skinner, F., 1975, "Designing a Multiple Prehension Manipulator," Mech. Eng. (Am. Soc. Mech. Eng.), September, pp. 30-37.

[3] Okada, T., 1979, "Computer Control of Multi-Jointed Finger System," Sixth International Joint Conference on Artificial Intelligence, Tokyo, Japan.

[4] Salisbury, J. K., 1982, "Kinematic and Force Analysis of Articulated Hands," Ph.D. thesis, Dept. of Mechanical Engineering, Stanford University, Stanford, CA.

[5] Jacobsen, S. C., Wood, J. E., Knutti, D. F., and Biggers, K. B., 1984, "The Utah/MIT Dexterous Hand," Int. J. Robot. Res., 3(4), pp. 21-50.

[6] Lin, L. R., and Huang, H. P., 1996, "Mechanism Design of a New Multifingered Robot Hand," IEEE International Conference on Robotics and Automation, April 21-27, Minneapolis, MN, pp. 1471-1476.

[7] Salisbury, J. K., and Roth, B., 1983, "Kinematic and Force Analysis of Articulated Mechanical Hands," ASME J. Mech., Transm., Autom. Des., 105(1), pp. $35-41$.

[8] Rouff, C. F., and Salisbury, J. K., 1990, "Multi-Fingered Robotic Hand," U.S Patent 4,921,293.

[9] Reuleaux, F., 1963, The Kinematics of Machinery, A. B. W. Kennedy, translator, Dover Publications, New York, NY.

[10] Lakshminarayana, K., 1978, "Mechanics of Form Closure," ASME, paper no. 78-DET-32.
[11] Hunt, K. H., 1978, Kinematic Geometry of Mechanisms, Oxford University Press, Oxford.

[12] Hunt, K. H., 1983, "Structural Kinematics of In-parallel-Actuated RobotArms," ASME J. Mech., Transm., Autom. Des., 105(4), pp. 705-712.

[13] Earl, C. F., and Rooney, J., 1983, "Some Kinematic Structures for Robot Manipulator Design,” ASME J. Mech., Transm., Autom. Des., 105(1), pp. $15-22$.

[14] Tasi, L. W., 1999, Robot Analysis: The Mechanics of Serial and Parallel Manipulators, Chap. 3, John Wiley \& Sons, New York, NY.

[15] Somov, P., 1897, "Über Schraubengeschwindigkeiten eines festen Körpers beiverschiedener Zahl von Stützflachen," Z. Angew. Math. Phys., 42, pp. 133 $153,161-182$

[16] Rimon, E., and Burdick, J., 1996, “On Force and Form Closure for Multiple Finger Grasps," Proceedings of the 1996 IEEE International Conference on Robotics and Automation pp. 1795-1800.

[17] Yoshikawa, T., 1996, "Passive and Active Closures by Constraining Mechanisms," Proceedings of the 1996 IEEE International Conference on Robotics and Automation, pp. 1477-1484.

[18] Goldman, A. J., and Tucker, A. W., 1956, Polyhedral Convex Cones. In Linear Inequalities and Related Systems, eds. H. W. Kuhn and A. W. Tucker. Princeton University Press.

[19] Vassura, G., and Nerozzi, A., 1980, “A Multi-Finger Gripper,” Proceedings of the 10th ISIR, Milan, Italy, pp. 215-223. 